University of Windsor

Scholarship at UWindsor

1992

\title{
Many-electron radial and angular integrals in the unitary-group approach
}

R. D. Kent

Gordon W. F. Drake

University of Windsor

Follow this and additional works at: https://scholar.uwindsor.ca/physicspub

Part of the Physics Commons

\section{Recommended Citation}

Kent, R. D. and Drake, Gordon W. F.. (1992). Many-electron radial and angular integrals in the unitary-group approach. Physical Review A, 45 (5), 3339-3342.

https://scholar.uwindsor.ca/physicspub/78

This Article is brought to you for free and open access by the Department of Physics at Scholarship at UWindsor. It has been accepted for inclusion in Physics Publications by an authorized administrator of Scholarship at UWindsor. For more information, please contact scholarship@uwindsor.ca. 


\title{
Many-electron radial and angular integrals in the unitary-group approach
}

\author{
R. D. Kent \\ School of Computer Science, The University of Windsor, Windsor, Ontario, Canada N9B 3P4 \\ M. Schlesinger and G. W. F. Drake \\ Department of Physics, The University of Windsor, Windsor, Ontario, Canada N9B 3P4
}

(Received 26 July 1991; revised manuscript received 9 September 1991)

\begin{abstract}
An extension of work by Drake [Phys. Rev. A 18, 820 (1978)] to the treatment of radial and angular integrals occurring in $\mathrm{N}$-electron systems is presented. It is shown that the essential results pertaining to two-electron systems adapted to Hylleraas coordinates also apply to many electrons when pairs of electrons are chosen to interact with each other in a spin-adapted basis set. These results are derived using graphical-analysis techniques in the context of the unitary-group approach.
\end{abstract}

PACS number(s): 31.15. $+\mathrm{q}$, 03.65.Fd, 02.20.+b

\section{INTRODUCTION}

The unitary-group approach (UGA) has been shown [1-4] to be particularly useful in a number of application areas, including configuration-interaction (CI) calculations [5], NMR [6], and SU(n)-based [7] treatments of many-particle problems. For such applications one must evaluate radial and angular integrals in the permutation symmetry-adapted $\left(S_{N}\right)$ many-electron basis. The $\mathrm{U}(n)$ basis, expressed alternatively in terms of Weyl-Young [3] (WYT) or Paldus [2] (ABC) tableaux, or Shavitt's [5] distinct row table (DRT), provides for considerable simplification of both spin-independent and spindependent operators and methods for evaluating their matrix elements.

In this paper we describe an approach to obtaining radial integral relations by reducing matrix elements of tensor operators that depend on the interparticle coordinates to finite sums of radial integrals for spin-adapted states of arbitrary angular momentum. This work is a natural extension of results obtained by Drake [8] for the twoelectron cases and reduces to his results in that limit.

The organization of the paper is as follows. In Sec. II we briefly review the two-electron theory. In Sec. III we deal with the cases of $N$ electrons where the system of particles is subdivided into two particles plus the remaining coupled system of $N-2$ electrons.

\section{TWO-ELECTRON SYSTEMS}

Drake [8] has shown that for two-electron systems described by Hylleraas coordinates $\mathbf{r}_{1}, \mathbf{r}_{2}$, and $\mathbf{r}_{12}=\mathbf{r}_{1}-\mathbf{r}_{2}$ $\left(r=\left|\mathbf{r}_{12}\right|\right)$, and whose wave functions are vector-coupled eigenfunctions of the total angular momentum operator $\mathbf{L}=l_{1}+l_{2}$, it is possible to derive a number of recurrence relations for the associated radial integrals. Further, by correlating the spins using the total spin operator $\mathbf{S}=\mathbf{s}_{1}+\mathbf{s}_{2}$ (basically, applying Pauli's principle), additional restrictions are placed on the allowed values of $L$, thereby simplifying the radial integral expressions even more.

The general problem can be stated in terms of evaluating integrals of the form

$$
\begin{aligned}
I\left(l_{1} m_{1}, l_{2} m_{2} ; R^{\prime} R\right) \iint & d \mathbf{r}_{1} d \mathbf{r}_{2} R^{\prime} Y_{l_{1}^{\prime} l_{2}^{\prime} L^{\prime}}^{M^{\prime}}\left(\hat{\mathbf{r}}_{1} \widehat{\mathbf{r}}_{2}\right)^{*} \\
& \times T_{k_{1} k_{2} K}^{Q} Y_{l_{1} l_{2} L}^{M}\left(\hat{\mathbf{r}}_{1}, \hat{\mathbf{r}}_{2}\right) R,
\end{aligned}
$$

where $Y_{l_{1}^{\prime} l_{2}^{\prime} L^{\prime \prime}}, \quad Y_{l_{1} l_{2} L}^{M}$ and $T_{k_{1} k_{2} K}^{Q}$ all denote vectorcoupled products of spherical harmonics (unit irreducible tensor operators) of the forms

$Y_{l_{1} l_{2} L}^{M}\left(\hat{\mathbf{r}}_{1}, \widehat{\mathbf{r}}_{2}\right)=\sum_{m_{1}, m_{2}}\left\langle l_{1} l_{2} m_{1} m_{2} \mid L M\right\rangle Y_{l_{1}}^{m_{1}}\left(\hat{\mathbf{r}}_{1}\right) Y_{l_{2}}^{m_{2}}\left(\hat{\mathbf{r}}_{2}\right)$

and

$T_{k_{1} k_{2} K}^{Q}\left(\hat{\mathbf{r}}_{1}, \hat{\mathbf{r}}_{2}\right)=\sum_{\mu_{1}, \mu_{2}}\left\langle k_{1} k_{2} \mu_{1} \mu_{2} \mid K Q\right\rangle Y_{k_{1}}^{\mu_{1}}\left(\hat{\mathbf{r}}_{1}\right) Y_{k_{2}}^{\mu_{2}}\left(\hat{\mathbf{r}}_{2}\right)$,

and $R=R\left(r_{1}, r_{2}, r\right)$ denotes the correlated radial part of the wave function. The volume element is expressed in the form $d \mathbf{r}_{1} d \mathbf{r}_{2}=r_{1} d r_{1} r_{2} d r_{2} r d r \sin \theta_{1} d \theta_{1} d \phi_{1} d \chi$ where $\theta_{1}$ and $\phi_{1}$ are the polar angles of the vector $r_{1}$, and $\chi$ is the angle of rotation of the rigid triangle formed by $r_{1}, r_{2}$, and $\mathbf{r}$ about the $\mathbf{r}_{1}$ direction.

The independent variables in the problem are $r_{1}, r_{2}, r$, $\theta_{1}, \phi_{1}$, and $\chi$. Using the rotation matrix relation, the spherical harmonic can be expressed as

$$
Y_{l_{2}}^{m_{2}}\left(\theta_{2}, \phi_{2}\right)=\sum_{m} D_{m_{2}, m}^{l_{2}}\left(\phi_{1}, \theta_{1}, \chi\right)^{*} Y_{l_{2}}^{m}(\theta, \phi),
$$

where $\theta$ and $\phi$ are the polar angles of $\mathbf{r}_{2}$ relative to $\mathbf{r}_{1}$. This leads to the fundamental integral relation

$$
I\left(l_{1} m_{1}, l_{2} m_{2} ; R R^{\prime}\right)=2 \pi \delta_{m_{1} m_{2}} \delta_{l_{1} l_{2}} I_{l_{1}}\left(R^{\prime} R\right),
$$

where

$$
I_{l}\left(R^{\prime} R\right)=\int_{0}^{\infty} r_{1} d r_{1} \int_{0}^{\infty} r_{2} d r_{2} \int_{\left|r_{1}-r_{2}\right|}^{r_{1}+r_{2}} r d r R^{\prime} R P_{1}(\cos \theta),
$$

and where $P_{1}(\cos \theta)$ is a Legendre polynomial and $\cos \theta$ is a (radial) function defined by

$$
\cos \theta=\left(r_{1}^{2}+r_{2}^{2}-r^{2}\right) / 2 r_{1} r_{2} .
$$

The full integral (1) can be expressed using standard angular momentum coupling techniques; hence 


$$
I=(-1)^{L^{\prime}-M^{\prime}}\left[\begin{array}{ccc}
L^{\prime} & K & L \\
-M^{\prime} & Q & M
\end{array} \sum_{\lambda_{1}, \lambda_{2}, \Lambda} X_{\lambda_{1}, \lambda_{2}, \Lambda} D_{\lambda_{1}, \lambda_{2}, \Lambda} I_{\Lambda}\left(R^{\prime} R\right)\right.
$$

where $I_{\Lambda}\left(R^{\prime} R\right)$ is expressed using (6) and the $X$ and $D$ factors are

$$
\begin{aligned}
X_{\lambda_{1}, \lambda_{2}, \Lambda}= & \frac{(-1)^{l_{1}^{\prime}+l_{2}^{\prime}+L^{\prime}+\Lambda}}{8 \pi}\left(\lambda_{1}, \lambda_{2}, \Lambda\right)\left(l_{1}, k_{1}, l_{1}^{\prime}, l_{2}, k_{2}, l_{2}^{\prime}, L, L^{\prime}, K\right)^{1 / 2} \\
& \times\left(\begin{array}{ccc}
l_{1} & k_{1} & \lambda_{1} \\
0 & 0 & 0
\end{array}\right]\left[\begin{array}{ccc}
l_{1}^{\prime} & \lambda_{1} & \Lambda \\
0 & 0 & 0
\end{array}\right]\left[\begin{array}{ccc}
l_{2}^{\prime} & k_{2} & \lambda_{2} \\
0 & 0 & 0
\end{array}\right]\left[\begin{array}{ccc}
l_{2}^{\prime} & \lambda_{2} & \Lambda \\
0 & 0 & 0
\end{array}\right]
\end{aligned}
$$

and

$$
D_{\lambda_{1}, \lambda_{2}, \Lambda}=\left\{\begin{array}{lll}
L^{\prime} & l_{2}^{\prime} & l_{1}^{\prime} \\
\Lambda & \lambda_{1} & \lambda_{2}
\end{array}\right\}\left\{\begin{array}{lll}
l_{1} & l_{2} & L \\
k_{1} & k_{2} & K \\
\lambda_{1} & \lambda_{2} & L^{\prime}
\end{array}\right\} .
$$

We use the standard notation $(a, b, \ldots)$ $=(2 a+1)(2 b+1) \ldots$ in Eq. (9). form

The above formulas allow for (1) to be expressed in the

$$
I=\sum_{\Lambda} C_{\Lambda} I_{\Lambda}\left(R^{\prime} R\right)
$$

where the $C_{\Lambda}$ are the angular coefficients

$$
C_{\Lambda}=\sum_{\lambda_{1}, \lambda_{2}} C_{\lambda_{1}, \lambda_{2}, \Lambda}
$$

and

$$
C_{\lambda_{1}, \lambda_{2}, \Lambda}=(-1)^{L^{\prime}-M^{\prime}}\left[\begin{array}{ccc}
L^{\prime} & K & L \\
-M^{\prime} & Q & M
\end{array}\right] X_{\lambda_{1}, \lambda_{2}, \Lambda} D_{\lambda_{1}, \lambda_{2}, \Lambda}
$$

The importance of the above analysis lies in the fact that the matrix elements for a wide variety of operators are reduced to sums of radial integrals of the form (6). For these integrals, recursion relations may be employed to further reduce the amount of computation.

\section{MANY-ELECTRON SYSTEMS}

As stated in the Introduction, our treatment amounts to viewing the system of $N$ electrons as made up of a pair of electrons interacting with all the rest. The need to remove two electrons at a time, rather than one, arises from an enumeration of the independent variables in the problem. Consider, for example, a three-electron system and take $r_{23}=\left|\mathbf{r}_{2}-\mathbf{r}_{3}\right|$ as an independent variable. The volume element is then $d \mathbf{r}_{1} d \mathbf{r}_{2} d \mathbf{r}_{3}$ $=d \mathbf{r}_{1} r_{2} d r_{2} r_{3} d r_{3} r_{23} d r_{23} \sin \theta_{2} d \theta_{2} d \phi_{2} d \chi$. If $\mathbf{r}_{2}$ is thought of as independent, then $r_{3}$ is partially determined by the values of $r_{23}$ and $\chi$. The coordinate $r_{23}$ now plays the role of $r$ in Sec. II. Each state adapted to total angular momentum $L$ is constructed by adapting to total spin $S$, following the UGA (see Ref. [3]), by coupling successive particles, $l_{k} m_{k}, k=1, \ldots, N$, in a manner consistent with the spin coupling.

The matrix elements of the tensor operator $T_{k_{1} k_{2} K}^{Q}\left(\hat{\mathbf{r}}_{i}, \widehat{\mathbf{r}}_{j}\right)$ in the $N$-electron system is determined by decoupling the $i$ th and $j$ th electrons and recoupling the remaining $N-2$ electrons into a new system (quasiparticle) with total angular momentum $\tilde{L}$. The $i$ th and $j$ th electrons are coupled to a two-electron state with angular momentum $\Gamma$. Equations (1), (2), and (3) are consequently to be modified by replacing $l_{1}, m_{1}, l_{2}, m_{2}, L$ and $M$ by $l_{i}, m_{i}, l_{j}, m_{j}, \Gamma$, and $M$ (and similarly for primed labels). The issue that remains, therefore, is to transform the original $N$-electron coupled state into the decoupled system consisting of a pair of electrons plus the $N-2$ electron subsystem; these are subsequently recoupled to a resultant state of total $L$ and to the tensor operator.

The process of decoupling and recoupling is accomplished using the techniques of group subduction [9], in general. However, since we need only consider paired states it is fully equivalent to vector coupling. For the $i$ th and $j$ th electrons in the bra state interacting with the $r$ th and $t$ th electron in the ket state, the equivalent expression to (8) now becomes

$$
\begin{aligned}
I\left(l_{i}, m_{i}, l_{j}, m_{j}, l_{r}^{\prime} m_{r}^{\prime}, l_{t}^{\prime}, m_{t}^{\prime}\right) \\
=(-1)^{\Gamma^{\prime}-M^{\prime}}\left(\begin{array}{ccc}
\Gamma^{\prime} & K & \Gamma \\
-M^{\prime} & Q & M
\end{array}\right) \\
\quad \times \sum_{\lambda_{1}, \lambda_{2}, \Lambda} \hat{X}_{\lambda_{1}, \lambda_{2}, \Lambda} \hat{D}_{\lambda_{1}, \lambda_{2}, \Lambda} I_{\Lambda}\left(R^{\prime} R\right)
\end{aligned}
$$

where $R=R\left(r_{1}, \ldots, r_{N}\right)$ and $R^{\prime}=R^{\prime}\left(r_{1}, \ldots, r_{N}\right)$ replace the previous correlated radial parts of the wave function. The factor $\hat{X}_{\lambda_{1}, \lambda_{2}, \Lambda}$ is expressed as

$\widehat{X}_{\lambda_{1}, \lambda_{2}, \Lambda}=\frac{(-1)^{l_{r}^{\prime}+l_{t}^{\prime}+\Gamma+\Lambda}}{8 \pi}\left(\lambda_{1}, \lambda_{2}, \Lambda\right)\left(l_{i}, k_{1}, l_{r}^{\prime}, l_{j}, k_{2}, l_{t}^{\prime}, \Gamma, \Gamma^{\prime}, K\right)^{1 / 2}\left(\begin{array}{ccc}l_{i} & k_{1} & \lambda_{1} \\ 0 & 0 & 0\end{array}\right)\left(\begin{array}{ccc}l_{r}^{\prime} & \lambda_{1} & \Lambda \\ 0 & 0 & 0\end{array}\right)\left(\begin{array}{ccc}l_{j} & k_{2} & \lambda_{2} \\ 0 & 0 & 0\end{array}\right]\left(\begin{array}{ccc}l_{t}^{\prime} & \lambda_{2} & \Lambda \\ 0 & 0 & 0\end{array}\right]$.

The factor $\hat{D}_{\lambda_{1}, \lambda_{2}, \Lambda}$ is represented by the angular momentum coupling graph shown in Fig. 1 . This graph can be decomposed by separating across sets of three lines [10], thereby expanding the subduction coefficients for bra and ket states in terms of $6-j$ coefficients as shown in Fig. 2, to arrive at the expression 


$$
\begin{aligned}
& \hat{D}_{\lambda_{1}, \lambda_{2}, \Lambda}=\prod_{k=r+1}^{t-1}\left(L_{k}^{\prime}, \widetilde{L}_{k-1}^{\prime}\right)^{1 / 2}(-1)^{\tilde{L}_{k-1}^{\prime}+L_{k+1}^{\prime}+l_{r}^{\prime}+l_{k+1}^{\prime}}\left\{\begin{array}{llc}
L_{k+1}^{\prime} & \tilde{L}_{k}^{\prime} & l_{k+1}^{\prime} \\
\widetilde{L}_{k-1}^{\prime} & L_{k}^{\prime} & l_{r}^{\prime}
\end{array}\right\}(-1)^{L_{t}^{\prime}+\tilde{L}_{t-2}^{\prime}+l_{t}^{\prime}+l_{r}^{\prime}}\left\{\begin{array}{ccc}
L_{t}^{\prime} & \Gamma^{\prime} & \tilde{L}_{t-2}^{\prime} \\
l_{r}^{\prime} & L_{t-1}^{\prime} & l_{t}^{\prime}
\end{array}\right\} \\
& \times \prod_{k=t+1}^{N-1}\left(L_{k}^{\prime}, \widetilde{L}_{k-2}^{\prime}\right)^{1 / 2}(-1)^{\tilde{L}_{k-2}^{\prime}+L_{k+1}^{\prime}+\Gamma^{\prime}+l_{k+1}^{\prime}}\left\{\begin{array}{ccc}
L_{k+1}^{\prime} & \widetilde{L}_{k-1}^{\prime} & l_{k+1}^{\prime} \\
\widetilde{L}_{k-2}^{\prime} & L_{k}^{\prime} & \Gamma^{\prime}
\end{array}\right\} \\
& \times \prod_{k=i+1}^{j-1}\left(L_{k}, \tilde{L}_{k-1}\right)^{1 / 2}(-1)^{L_{k+1}+\tilde{L}_{k-1}+l_{i}+l_{k+1}}\left\{\begin{array}{lll}
\tilde{L}_{k} & L_{k+1} & l_{k+1} \\
L_{k} & \tilde{L}_{k-1} & l_{i}
\end{array}\right\} \\
& \times(-1)^{L_{j}+\tilde{L}_{j-2}+l_{i}+l_{j}}\left\{\begin{array}{ccc}
L_{j-1} & l_{i} & \widetilde{L}_{j-2} \\
\Gamma & L_{j} & l_{j}
\end{array}\right\} \\
& \times \prod_{k=j+1}^{N-1}\left(L_{k}, \tilde{L}_{k-2}\right)^{1 / 2}(-1)^{L_{k+1}+\tilde{L}_{k-2}+\Gamma+1_{k+1}}\left\{\begin{array}{ccc}
\widetilde{L}_{k-1} & L_{k+1} & l_{k} \\
L_{k} & \widetilde{L}_{k-2} & \Gamma
\end{array}\right\} \\
& \times(-1)^{L_{N}+\tilde{L}_{N-2}+\Gamma+L_{N}^{\prime}+\tilde{L}_{N-2}^{\prime}+\Gamma^{\prime}}\left\{\begin{array}{lll}
\Gamma^{\prime} & l_{t}^{\prime} & l_{r}^{\prime} \\
\Lambda & \lambda_{1} & \lambda_{2}
\end{array}\right\}\left\{\begin{array}{lll}
l_{i} & l_{j} & \Gamma \\
k_{1} & k_{2} & K \\
\lambda_{1} & \lambda_{2} & \Gamma^{\prime}
\end{array}\right\}
\end{aligned}
$$

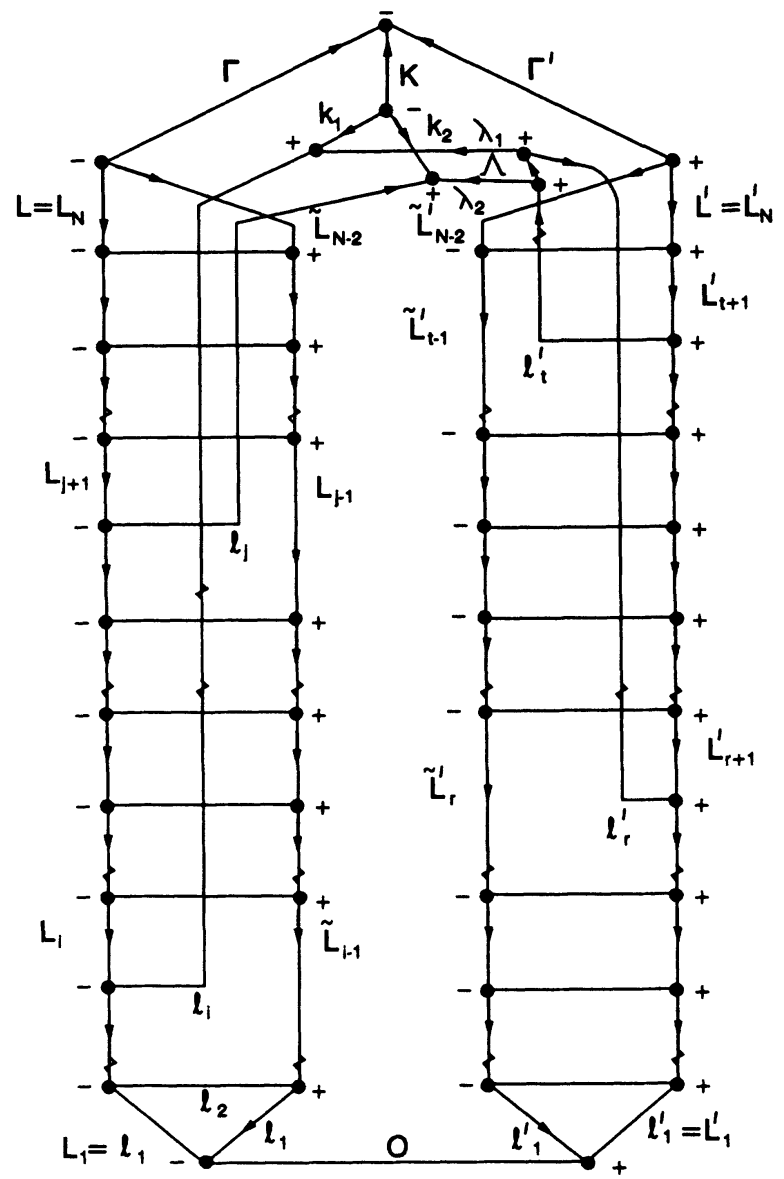

FIG. 1. Angular momentum coupling graph from Eq. (16) representing the $\widehat{D}_{\lambda_{1}, \lambda_{2}, \Lambda}$ coefficient. All unmarked lines are labeled with the appropriate single-particle angular momentum, $L_{k}, L_{k}^{\prime}, l_{k}, l_{k}^{\prime}, \widetilde{L}_{k}$, or $\widetilde{L}_{k}^{\prime}$ accordingly, and all angular momenta are assumed to be integers.
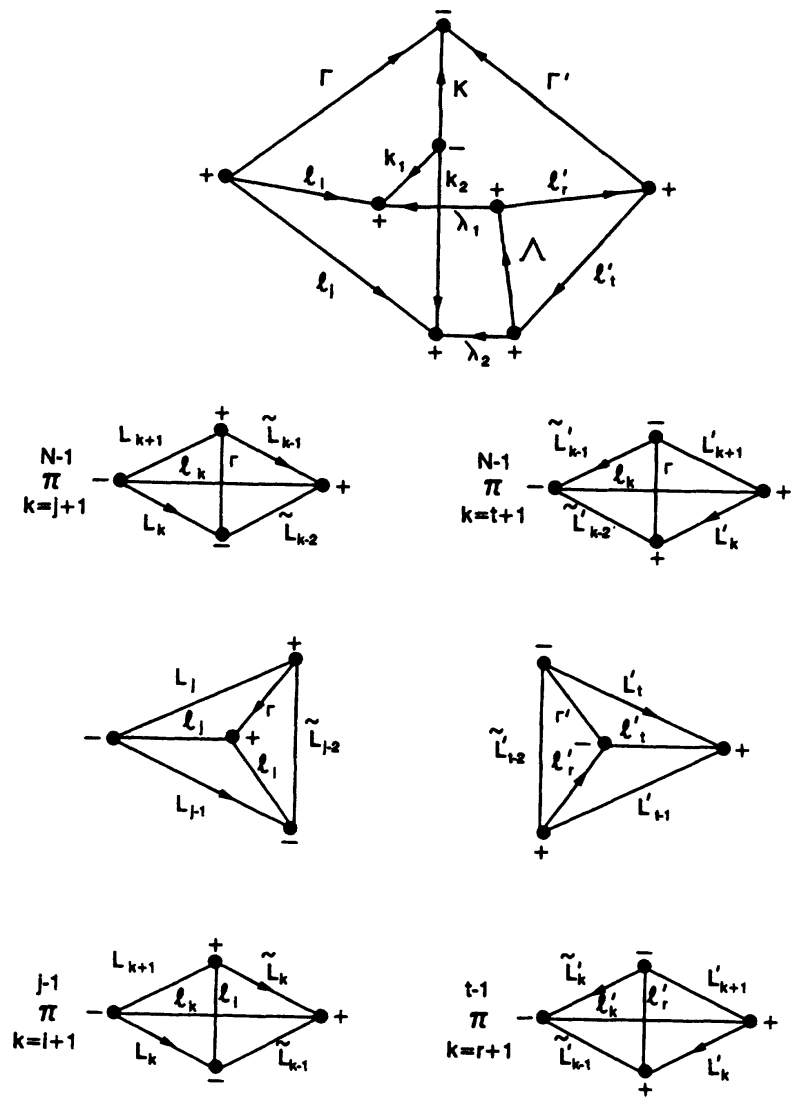

FIG. 2. Decomposition of the $\widehat{D}_{\lambda_{1}, \lambda_{2}, \Lambda}$ coefficient in terms of the product of the $D_{\lambda_{1}, \lambda_{2}, \Lambda}$ coefficient and subduction coefficients for recoupled bra and ket. The lines labeled by $\Gamma$ and $\Gamma^{\prime}$ represent paired-state angular momenta produced by the decoupling of the $(N-2)$-electron systems from the parent $N$ electron systems. 
In particular, the last product of $6-j$ and $9-j$ symbols derives from the top graph in Fig. 2 and corresponds to Fig. 1 of Ref. [8]. In the limit of $N=2$ electrons, the above results reduce to those of Drake.

In this case, as in the two-electron case, spin correlation, as provided for by the UGA $[11,12]$, greatly restricts the allowed $\widetilde{L}$ and $\Lambda$ values. Further, since the angular integration performed over all coordinates except $i, j, r$, and $t$ does not involve the action of the tensor operator, it follows that $\widetilde{L}_{k}=\widetilde{L}_{k}^{\prime}$ for all $k=1, \ldots, N-2$. The approach outlined above obviates the need to explicitly $L$ adapt linear combinations of WYT, as shown, for instance, in Ref. [13].

\section{CONCLUSION}

We have shown that it is possible to extend Drake's treatment of two-electron radial integral techniques using Hylleraas coordinates to many-electron systems. In do- ing so we have employed the interpretation of the $\mathrm{N}$ particle system as a composite of a pair of electrons and an $(N-2)$-particle subsystem. Starting from the $N$ particle $\mathrm{U}(n)$ basis we first transformed to a decoupled pair plus an $(N-2)$-electron system requiring evaluation of subduction coefficients and then recoupled all angular momenta to the interaction tensor angular momenta. Since the basis states are spin adapted, the resultant factors are relatively simple to calculate and are few in number. We are currently engaged in obtaining more detailed results regarding recursion relations for various forms of tensor operators and plan to report on this in due course.

\section{ACKNOWLEDGMENT}

Research support by the Natural Sciences and Engineering Research Council of Canada is gratefully acknowledged.
[1] W. G. Harter, Phys. Rev. A 8, 2819 (1973).

[2] J. Paldus, J. Chem. Phys. 61, 5321 (1974).

[3] G. W. F. Drake and M. Schlesinger, Phys. Rev. A 15, 1990 (1977).

[4] The Unitary Group for the Evaluation of Electronic Energy Matrix Elements, edited by J. Hinze, Lecture Notes in Chemistry Vol. 22 (Springer-Verlag, Berlin, 1981). See, also, the many references contained therein.

[5] I. Shavitt, Mathematical Frontiers in Computational Chemical Physics, edited by D. G. Truhlar (SpringerVerlag, Berlin, 1988).

[6] R. D. Kent, M. Schlesinger, and Pardu S. Ponnapalli, Phys. Rev. A 39, 19 (1989); 39, 3260 (1989).
[7] R. D. Kent and M. Schlesinger, J. Math. Phys. 32, 1102 (1991).

[8] G. W. F. Drake, Phys. Rev. A 18, 820 (1978).

[9] R. D. Kent and M. Schlesinger, Phys. Rev. A 23, 979 (1981).

[10] D. M. Brink and G. R. Satchler, Angular Momentum, 2nd ed. (Clarendon, Oxford, 1968).

[11] R. D. Kent and M. Schlesinger, Phys. Rev. A 42, 1155 (1990).

[12] R. D. Kent, M. Schlesinger, and G. W. F. Drake, J. Comput. Phys. 40, 430 (1981).

[13] M. Schlesinger, R. D. Kent, and G. W. F. Drake, Can. J. Phys. 60, 357 (1982). 